\title{
Evaluation des effets des doses de mycorhizes sur les paramètres de croissance et de la production de trois variétés de Voandzou dans la localité de Dschang, Ouest Cameroun
}

\author{
Wang-Bara Bertrand \\ Institut de Recherche Agricole pour le Développement (IRAD), Station \\ Polyvalente de Recherche Agricole de Garoua SPRA-Garoua, Cameroun. \\ Université de Dschang, Faculté d'Agronomie et des Sciences Agricoles, \\ Dschang, Cameroun

\section{Amedep David} \\ Département des Sciences Biologiques, \\ Université de Ngaoundéré, Cameroun
}

\section{Housseini Djida Jacques}

Institut de Recherche Agricole pour le Développement (IRAD), Station Polyvalente de Recherche Agricole de Garoua SPRA-Garoua, Cameroun.

Département des Sciences Biologiques,

Université de Ngaoundéré, Cameroun

\section{Mana Gaston Guiwa}

Institut de Recherche Agricole pour le Développement (IRAD), Station

Polyvalente de Recherche Agricole de Garoua SPRA-Garoua, Cameroun

\section{Doi:10.19044/esj.2021.v17n17p213}

Submitted: 12 January 2021

Accepted: 19 February 2021

Published: 31 May 2021
Copyright 2021 Author(s)

Under Creative Commons BY-NC-ND

4.0 OPEN ACCESS

Cite As:

Bertrand W-B., David A., Jacques H.D. \& Guiwa M.G. (2021). Evaluation des effets des doses de mycorhizes sur les paramètres de croissance et de la production de trois variétés de Voandzou dans la localité de Dschang, Ouest Cameroun. European Scientific Journal, ESJ, 17(17), 213. https://doi.org/10.19044/esj.2021.v17n17p213

\section{Résumé}

Cette étude a pour objectif d'évaluer l'effet des doses d'un biofertilisant sur la croissance et la production du Voandzou à l'Ouest Cameroun. Elle a été menée à la Ferme d'Application et de la Recherche (FAR) et au Laboratoire de Phytopathologie et de Zoologie Appliquée de la FASA (Dschang) de Décembre 2017 à Juillet 2018. Pour cette étude trois variétés de Voandzou ont été utilisées, une variété locale de Dschang (VL) et deux autres 
variétés du Nord Cameroun (Mendéo V1M et Mendéo V2M). Deux doses de mycorhizes $(\mathrm{D} 1=10 \mathrm{~g}$ et $\mathrm{D} 2=20 \mathrm{~g})$ ont été inoculées et comparées au témoin (D0 $=0 \mathrm{~g}$ ) dans un dispositif en split-plot combinant deux facteurs (doses et variétés) avec 3 répétitions. Après avoir évalué le taux de germination des graines des différentes variétés, nous avons évalué les paramètres de croissance, la précocité variétale et la productivité en gousse des différentes variétés. Nos résultats ont montré que les variétés Mendéo 1 et 2 du Nord sont précoces avec une floraison débutant à partir de $40^{\mathrm{e}} \mathrm{JAS}$ et une maturité dès le $122^{\mathrm{e}} \mathrm{JAS}$. La variété VL est tardive avec une floraison dès le $58^{\mathrm{e}} \mathrm{JAS}$ et une maturité à $160^{\mathrm{e}} \mathrm{JAS}$. En général, les deux doses de mycorhize inoculées augmentent la croissance des plants de voandzou. Cependant la dose D2 (20g) semble être efficace pour les variétés Mendéo V1M et Mendéo V2M, par apport à la variété local VL qui préfère la dose D1 (10g). Le nombre de feuilles des trois variétés de Voandzou a été plus prononcé pour les plantes inoculées par rapport à celles non inoculées. La dose D2 (20g) d'inoculum a eu un effet positif sur la densité du feuillage des plantes des variétés V1M et V2M. Par contre, la variété locale (VL) de l'Ouest Cameroun parait plus favorable aux deux différentes doses (D1 et D2) de mycorhizes. Cette étude a montré que l'inoculation directe des CMA pendant le semis entraine une augmentation du rendement en gousse de la VL et améliore la production de la variété introduite V1M. L'étude permet de conclure que la variété améliorée V1M et locale VL sont potentiellement aptes dans la zone à dose $20 \mathrm{~g}$.

Mots clés : Inoculation, Voandzou, Doses de mycorhizes, Variétés, Rendement, Ouest Cameroun 


\title{
Evaluation of Mycorrhiza Doses Effect on Growth Parametric and Production of 3 Varieties of Bambara Groundnuts on the Locality of Dschang, West Cameroon
}

\author{
Wang-Bara Bertrand \\ Institut de Recherche Agricole pour le Développement (IRAD), Station \\ Polyvalente de Recherche Agricole de Garoua SPRA-Garoua, Cameroun. \\ Université de Dschang, Faculté d'Agronomie et des Sciences Agricoles, \\ Dschang, Cameroun \\ Amedep David \\ Département des Sciences Biologiques, \\ Université de Ngaoundéré, Cameroun \\ Housseini Djida Jacques \\ Institut de Recherche Agricole pour le Développement (IRAD), Station \\ Polyvalente de Recherche Agricole de Garoua SPRA-Garoua, Cameroun. \\ Département des Sciences Biologiques, \\ Université de Ngaoundéré, Cameroun \\ Mana Gaston Guiwa \\ Institut de Recherche Agricole pour le Développement (IRAD), Station \\ Polyvalente de Recherche Agricole de Garoua SPRA-Garoua, Cameroun
}

Abstract
Aim of this study is to evaluate the effect of bio-fertilizers on growth
and production of Bambara groundnut in West Cameroon. The study was
carried in the Experimental and Research Farm and in the Phytopathology
Laboratory and Applied Zoology of FAAS. For this study, 3 varieties of
Bambara groundnuts have been used, the Local varieties of Dschang (VL) and
two varieties of North Cameroon (Mendé V1M et Mendé V2M). Two doses
of mycorrhiza at 10 and 20 g were compared with the control (0 g) in a split-
plot design combining two factors (doses and varieties) with 3 repetitions.
After determination of germination rate of seeds for each different variety, we
had determined agronomic performances such as the growth of plants,
precocity and yield pods. The results were proved that the two varieties of
North Cameroon are early varieties with an average flowering of 40 DAS and
a maturity of 122 DAS. The local variety VL is a late variety with a flowering
of 58 DAS and a maturity of 160 DAS. The two doses of mycorrhiza at 10
(D1) and $20 \mathrm{~g}$ (D2) increases plants growth of Bambara groundnuts. However,
the doses of mycorrhiza at $20 \mathrm{~g}$ appear favorable for the two varieties of North
(Mendéo V1M and Mendéo V2M) compared to the variety of West which is
favorable at the dose of $10 \mathrm{~g}$. The number of leaves from the three varieties of 
Bambara groundnuts had been improved from inoculated plants compared to non inoculated plants. The dose of $20 \mathrm{~g}$ has a positive effect on leaves density of varieties V1M and V2M. In contrast, the Local variety of West Cameroon appear favorable both two different doses (D1 and D2) of mycorrhiza. This study had showed that the direct inoculation with Fungi Arbuscular Mycorrhiza increase yield pods of Local variety (VL) of West Cameroon and improve the production of variety V1M of Nord Cameroon. Study permits to conclude that the variety V2M of North and V1L of West Cameroon are able potentially on the study zone at $20 \mathrm{~g}$.

Keywords: Inoculation, Bambara groundnut, Doses of mycorhiza, Varieties, Yields, West Cameroon

\section{Introduction}

De nombreux pays africains ont investi dans la production des cultures de rentes (Café et Cacao) et surtout industrielles au détriment des cultures vivrières dont certaines sont à l'abandon et menacées de disparition comme les plantes alimentaires (Djè et al., 2005). Les légumineuses à graines, bien qu'étant des plantes alimentaires marginales, jouent un rôle important dans l'amélioration des propriétés des sols, en augmentant le rendement des cultures non fixatrices d'azotes en association ou rotation (Bado, 2002). Biologiquement, elles ont la capacité à fixer l'azote atmosphérique et de fertiliser le sol grâce à la présence des nodosités racinaires hébergeant des colonies de Rhizobium, bactéries symbiotiques (Rapport projet C2D, 2013 ; Amadou et al., 2018). Elles sont d'une grande importance pour les populations qui la cultivent sur le plan social, culturel et $75 \%$ de la nourriture de base proviennent des céréales et des légumineuses (Rapport projet C2D, 2013).

Le voandzou (Vigna subterranea (L.) Verdcout.), encore appelé pois bambara ou pois de terre, est une légumineuse alimentaire mineure dont la culture est restée dans les limites de l'Afrique sub-saharienne où il est adapté à diverses conditions climatiques et écologiques des zones agro-écologiques (BAMNET, 2000 ; Madou et al., 2018). Il est considéré en Afrique comme la troisième légumineuse la plus importante en termes de production et de consommation après l'arachide (Arachis hypogeae (L.)) et le niébé (Vigna unguiculata (L.))(Oparaeke et Bunmi, 2006). En Afrique de l'Ouest, où plus de $70 \%$ de la production mondiale totale est cultivée, il est devenu une partie intégrante des systèmes agricoles. Les graines servent à l'alimentation humaine, seule ou mélangée à d'autres aliments (Nacoulma-Ouédraogo, 1996). Les feuilles, riches en phosphore, servent à l'alimentation du bétail. Les graines et les feuilles sont utilisées dans la médecine traditionnelle (NacoulmaOuédraogo, 1996). Le voandzou est une plante hautement calorique (387 $\left.\mathrm{kcal} .100 \mathrm{~g}^{-1}\right)$, riche en vitamines et en éléments minéraux et très équilibrée en 
terme de protéine végétale (Oniwamo et al., 1998 ; Diallo et al., 2015 Minka et al., 2000 ; Madou et al., 2018). Sa culture contribue fortement à la résilience des populations à l'insécurité alimentaire et nutritionnelle (Ouoba et al., 2018 ). Le voandzou joue un rôle sur l'augmentation de la biodisponibilité du phosphore même dans les sols ferralitiques par le biais de sa capacité de fixation de l'azote (Andriamananjara, 2011). Le rendement du voandzou est réduit par une trop forte humidité du sol. Toutefois, les rendements moyens se situent entre 350 à $800 \mathrm{~kg} \cdot \mathrm{ha}^{-1}$ dans les régions où le sol est pauvre et la pluviométrie faible (Linnemann, 1995).

Au cours des dernières décennies, le phénomène de la dégradation des sols agricoles est devenu un problème mondial significatif (Mekuriaw et al., 2017). Étant une ressource limitée et finie pour laquelle il faut entre 200 à 1000 années pour la formation d'une couche de $2,5 \mathrm{~cm}$ d'épaisseur, son exploitation à des fins agricoles est fragilisé par l'augmentation de la population mondiale et le changement climatique (Ciampalini et al., 2011 ; Pimentel et al., 1995 cité par Moges et Taye, 2016). Dans le Hauts Plateaux de l'Ouest, la culture du voandzou jadis très répandue est en déclin sans raison agronomique apparente en dehors des tabous qui la considèrent comme une culture des femmes et aussi dépendante des coutumes. Il reste cependant cultivé de façon marginale dans plusieurs localités et consommé surtout en grains (bouilli ou grillé). Les travaux de recherche que ce soit à travers l'optimisation de l'efficience du travail cultural et de l'utilisation des biofertilisants efficaces, ont montrées que cette denrée peu onéreuse, accessible et sous-exploitée aide cette culture à mieux prélever les éléments nutritifs. Des mesures d'amélioration de la productivité sont envisageables pour booster le rendement des plantes à l'instar de l'utilisation des pesticides, d'engrais chimiques et des variétés améliorées de plantes (Dalgaard et al., 2003). Cette méthode reste toutefois dangereuse pour la santé du consommateur et l'environnement (Bowers et al., 2001). Toutefois, une amélioration de la fertilité des sols par l'utilisation de nouvelles stratégies est nécessaire. C'est dans ce contexte que le présent travail se propose d'étudier l'effet d'un biostimulant sur trois variétés de Voandzou en milieu paysan. L'objectif de cette étude est d'évaluer l'effet des doses à base de quatre souches de Champignon Mycorhizien Arbusculaire (CMA) sur la production de 3 variétés de Voandzou en champ. Les variétés introduites étaient celle Mendéo V1M et V2M du Nord et VL celle Locale de Dschang.

\section{I- $\quad$ Matériels et méthodes}

\section{I.1. Présentation de la zone d'étude}

Les travaux ont été réalisés dans la région de l'Ouest-Cameroun, plus précisément à la Ferme d'Application et de la Recherche (FAR) et au laboratoire de Phytopathologie et de Zoologie Appliquée de la FASA. Cette 
région d'une superficie de $13872 \mathrm{~km}^{2}$ est composée de 8 Départements (Manga et al., 2013). Elle a un climat de type tropical soudanien avec 2 grandes saisons: une saison sèche qui va de Octobre-Novembre à Mars-Avril et une saison de pluie qui commence en Mars-Avril jusqu'en OctobreNovembre. L'essai a été réalisé durant la période allant de décembre 2017 à Mai 2018. Durant cette période sèche, la température était de l'ordre de $21,11^{\circ}$ $\mathrm{C}$ allant jusqu'au mois de février, tandis que la pluviométrie partant de mifévrier à Mai était de $11,6 \mathrm{~mm}$. Elle se situe entre $5^{\circ} 0^{\prime} 6^{\circ} 0^{\prime}$ de latitude Nord et entre $10^{\circ} 0^{\prime} 11^{\circ} 0^{\prime}$ de longitude Est avec des températures variant entre 15 et $30^{\circ} \mathrm{C}$ soit une moyenne journalière de $25^{\circ} \mathrm{C}$.

\section{I.2 Situation agro-écologique}

Le site était couvert d'une courte jachère d'environ cinq mois, dominé par les graminées. Les influences anthropiques diverses ont contribué à la propagation d'espèces végétales diverses. Le paysage comprend les cultures (Maïs, Macabo, Légumes, Bananier plantain, Haricot), les jachères récentes, les étangs piscicoles, les bureaux de la Ferme d'Application et de la Recherche (FAR). Quelques espèces végétales rencontrées dans le site sont entre autres : Tithonia diversifolia, Mimosa pudica, Ageratum conyzoides, Cyperus esculentus, Bidens pilosa, Cynodon dactylon. Les précédents culturaux étaient une monoculture de Maïs (Zea mays). Le climat de la région de l'Ouest Cameroun est de type camerounien d'altitude. Il est caractérisé par une saison pluvieuse qui va de mi-mars à mi-novembre et une saison sèche qui va de minovembre à mi-mars. La pluviométrie moyenne annuelle varie entre 1800 et $2000 \mathrm{~mm}$ (IRAD, 2002).

\section{I.3 Caractéristiques climatiques de la région d'étude}

La Figure 1 montre la hauteur des précipitations enregistrée pendant la période de l'essai du Mois de Février à Mai 2018. D'après ce graphe, la hauteur des précipitations a varié en fonction des Mois. On a une fluctuation constante avec une valeur moyenne de 10,62 mm en début de saison pour les Mois de Février et Mars (fin saison sèche). Toutefois du Mois d'avril à Mai, on a une élévation de la hauteur des précipitations moyenne variant de 12,91 $\mathrm{mm}$ à 12,26 $\mathrm{mm}$ au mois d'Avril et Mai, traduisant le début de la saison pluvieuse. Plus on tend vers la saison effective de pluie, on a une élévation de la quantité d'eau enregistrée. 


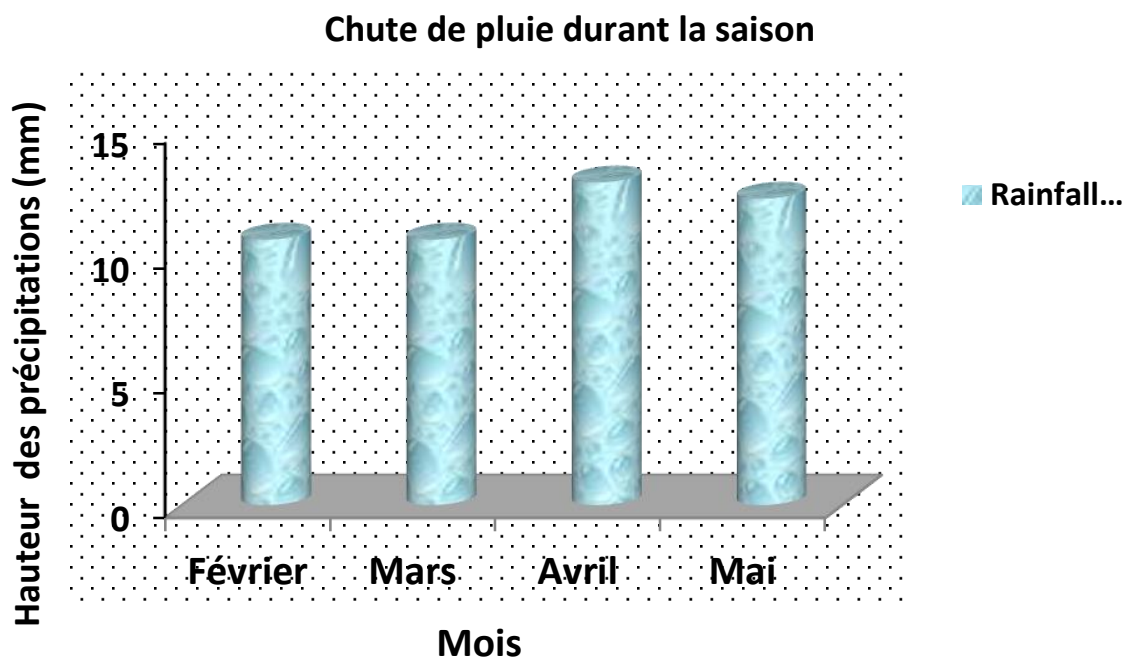

Figure 1 : Chute de pluie pendant la période de l'essai

Les données pluviométriques enregistrées pendant l'essai de Février à Mai 2018 sont illustrés sur le graphe 2. On constate selon le graphe une variation du volume d'eau reçu en fonction du Mois. Elle est constante avec une valeur moyenne de 58,88 ml en début de saison pour les Mois de Février et Mars. Par contre du Mois d'Avril à Mai, on a une augmentation du volume d'eau de pluie variant respectivement de 71,58 à $68 \mathrm{ml}$ selon la quantité d'eau reçue. Ce qui traduisant le début de la saison des pluies. Plus on tend vers la saison effective des pluies, on a une augmentation de la quantité d'eau de pluie.

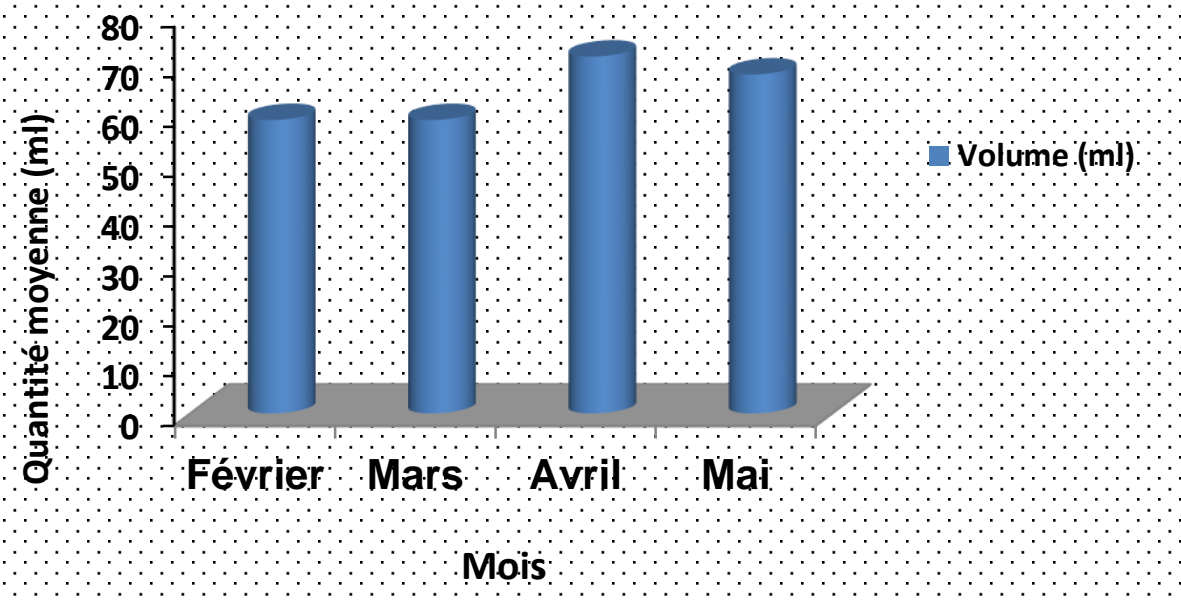

Figure 2 : Volume d'eau durant la période de l'essai 


\section{Matériels \\ II.1 Matériels végétal}

Dans cet essai, trois morphotypes de voandzou ont été utilisé, deux variétés améliorées du Nord à savoir : les variétés Mendéo blanche crème (V2M) et blanche beige (V1M) en raison de $03 \mathrm{~kg}$ chacune fournie par l'IRAD de Garoua en collaboration avec la SODECOTON et une variété locale brune claire de Dschang (VL), communément appelée « Matobô ou Nchoû » obtenue sur les marchés $(03 \mathrm{~kg})$. Les semences probablement infestées par l'association de mélange de plusieurs écotypes en stockage ont été séparée uniformément.

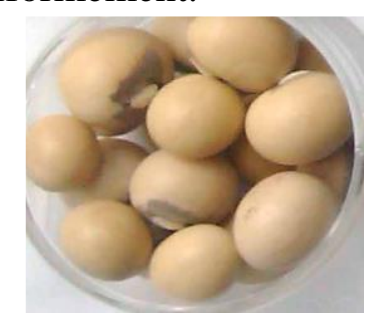

Nord (V1M)

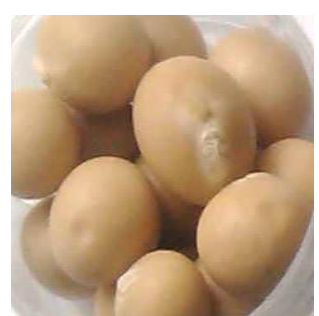

Nord (V2M)

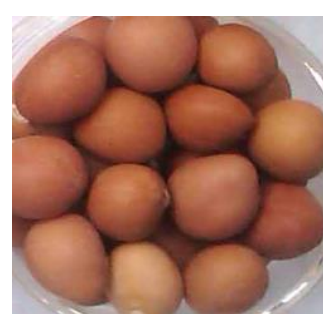

Dschang (VL)

Photo 1: Morphotypes de Voandzou utilisés (source, WANG-BARA Bertrand, 2018)

\section{II.2 Matériel biologique}

\section{II.2.1 Les Mycorhizes}

Le matériel biologique utilisé est celui du Bio-fertilisant Myco provenant du GIC AGRIBIO CAM issus des sols locaux et adapté pour les cultures en pépinières (maraîchage, fruitiers, plantes horticoles), en champ (céréales, légumineuses, fruitiers), les plantes médicinales, les plantes agroforestières et pâturages. Ce bio-fertilisant est constitué de 04 souches des champignons mycorhiziens à savoir : Rhizophagus irregularis (50\%), Scuttellospora gregaria (10\%), Gigaspora margarita (20\%), Glomus hoi (20 $\%)$.

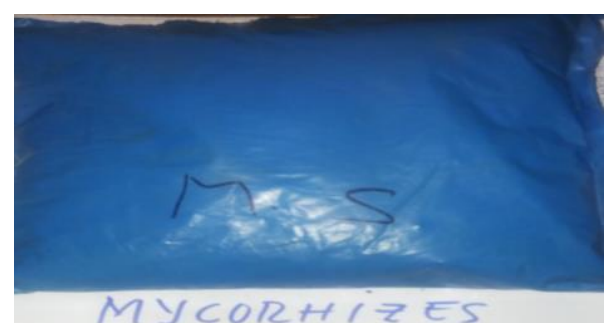

Photo 2 : Mycorhizes contenant les quatre souches de CMA (source, WANG-BARA Bertrand, 2018) 


\section{Méthodes}

\section{III.1 Dispositif expérimental et suivi de l'essai}

Le dispositif expérimental utilisé est un split-plot à 3 répétitions. L'essai portait sur deux facteurs à savoir : le facteur mycorhize et le facteur variété.

- Facteur en parcelle principal : les doses de mycorhize comprenant 03 modalités $(\mathrm{d} 0=$ témoin non inoculé, $\mathrm{d} 1=$ reçu $10 \mathrm{~g}$ de mycorhizes et $\mathrm{d} 2=$ reçu $20 \mathrm{~g}$ de mycorhizes).

- Facteur en parcelle secondaire : les variétés comprenant 03 modalités notamment la variété VL (Locale de Dschang), la variété V1 (Mendéo du Nord) et la variété V2 (Mendéo).

Chaque bloc comportait 3 sous-blocs subdivisés en 3 unités expérimentales (UE) chacun. Ce dispositif expérimental avait 27 UE au total d'où 9 UE par bloc. La superficie totale du dispositif expérimental était de 230 $\mathrm{m}^{2}$. Les UE étaient séparées les unes des autres de $0,5 \mathrm{~m}$. Les blocs qu'en à eux étaient séparé d'un mètre. Les unités expérimentales (UE) comportaient chacune 6 plantes sur la largeur et 8 plantes sur sa longueur à une densité de $25 \mathrm{~cm}$ x $35 \mathrm{~cm}$ d'où 48 plants par UE de $6 \mathrm{~m}^{2}$ de superficie. Cette densité nous permet d'avoir à moyenne 133.333 plants/ha. La planche 1 ci-dessous présente le dispositif expérimental de l'essai.

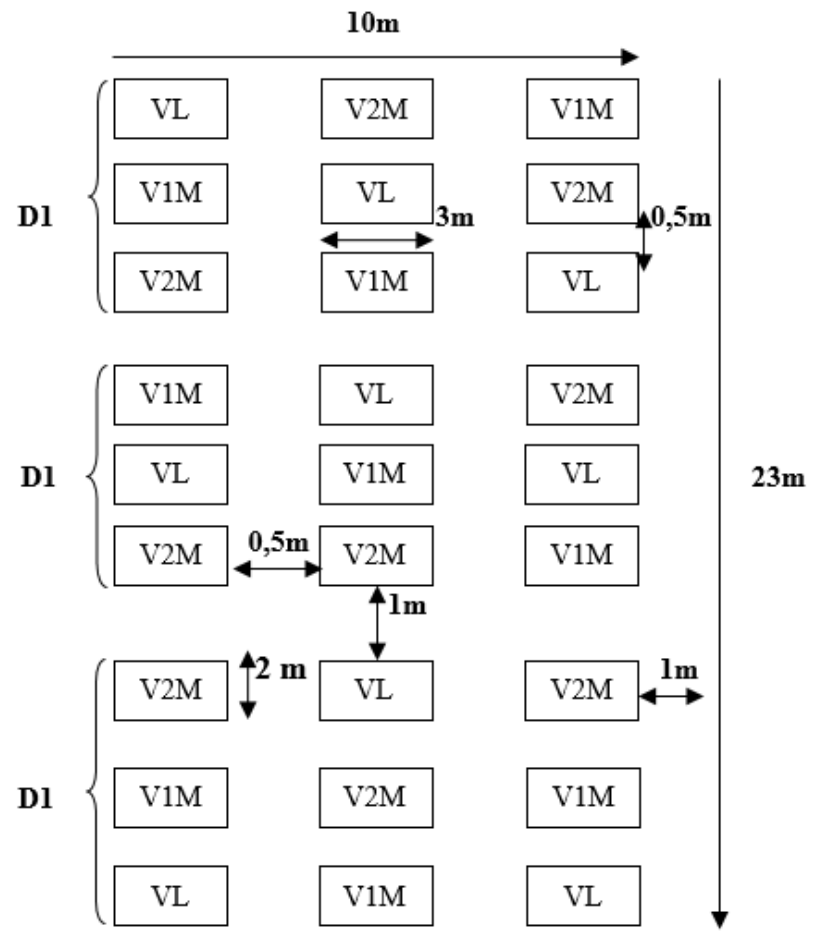

Planche 1 : Dispositif expérimental 


\section{III.2 Conduite de l'essai \\ III.2.1 Choix du site expérimental}

Le précédant cultural du site était une culture de Maïs réputée pour une bonne rotation de culture avec les légumineuses. L'essai a débuté par le désherbage, le labour de 15 à $20 \mathrm{~cm}$ de profondeur, la confection des UE et un arrosage constant de chaque unité expérimentale pendant une semaine ont été effectué.

\section{III.2.2 Semis par inoculation}

Le semis par inoculation a été réalisé en contre saison. Les graines ont été semées le 27 décembre 2017 sur un sol labouré et remué en raison de deux graines par poquet à une profondeur de 02 à $03 \mathrm{~cm}$. La méthode d'inoculation appliquée est celle recommandée par les fabricants (GIC AGRO-BIOCAM). Selon cette méthode, elle s'effectue lors du semis direct des morphotypes consistant à appliquer les différentes doses d'inoculum mycorhizien $(0 \mathrm{~g}, 10$ $\mathrm{g}$ et $20 \mathrm{~g}$ ) en dessous de la graine dans chaque poquet de semis du site expérimental. Les témoins non mycorhizés ont été premièrement semés pour éviter l'effet de contamination des bio-fertilisants.

\section{III.2.3 Apport d'eau et entretien}

Après la mise en place de l'essai, des travaux d'entretien ont été réalisés directement en débutant par l'arrosage des unités expérimentales, pour favoriser la germination des semences. Par la suite, la fréquence d'arrosage a été définie en raison de 5 jours par semaine, soit 4 arrosoirs par unité expérimentale, car le rendement du voandzou est réduit par une trop forte humidité du sol et cette plante est tolérante au stress hydrique. Trois désherbages ont été réalisés en vue de limiter la concurrence avec les mauvaises herbes. Le premier a eu lieu 15 jours après le semis, le second à 30 jours et le troisième à 45 jours après semis. Pour ce qui est de l'apport d'eau durant la phase de croissance des plantes pendant la saison sèche, il consiste à faire un supplément d'eau par arrosage des unités en matinée et en soirée. Pour assurer le bon développement de la culture en place, un apport moyen de $44 \mathrm{~L}$ d'eau journalier était effectué chaque jour sur toutes les unités expérimentales jusqu'à la phase de la floraison des plantes. Concernant le reste du cycle de développement de la plante (maturation des gousses), l'apport en eau a été assuré par la reprise de la saison de pluie. 

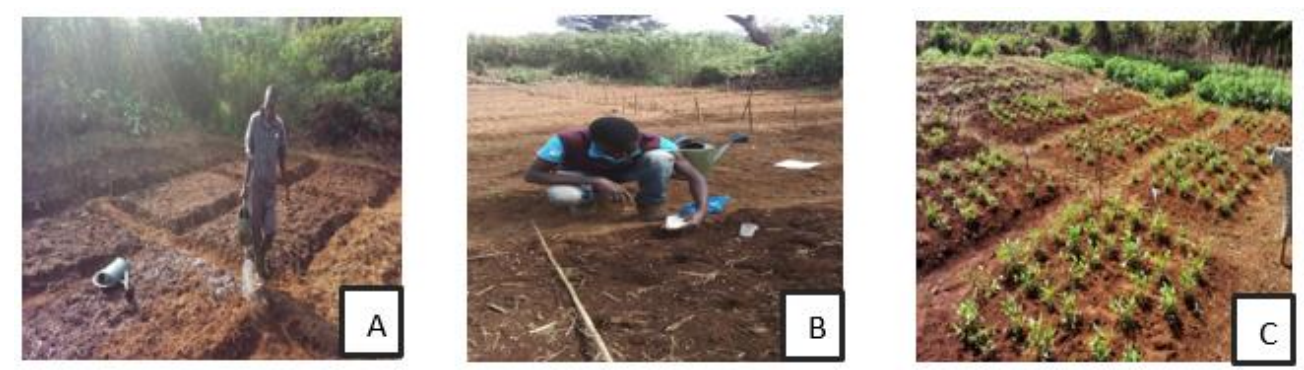

Picture 3 : Confection et arrosage (A), semis par inoculation (B) et entretien des plants (C)

\section{III.2.4 Récolte}

La récolte a été effectuée après que les différents cultivars aient atteint leur maturité. Elle a été effectuée lorsqu'environ $70 \%$ des plantes d'une unité élémentaire présentaient des gousses ayant atteints le stade mature (gousses remplies et chaque graine ayant sa couleur spécifique du morphotype). La récolte s'est déroulée entre $120^{\mathrm{e}}$ et $121^{\mathrm{e}} \mathrm{JAS}$ pour les deux variétés Mendéo du Nord. Par contre pour la variété Locale de Dschang, la récolte s'est déroulée à partir du $160^{\mathrm{e}} \mathrm{JAS}$.

\section{III.3 Evaluation des paramètres}

Au cours de cette phase végétative cinq variables ont été pris en compte : le taux de germination (au laboratoire), la hauteur des plantes $(\mathrm{cm})$, le nombre de feuilles (trifoliolées), la précocité variétale et le rendement en gousse des variétés.

\section{III.3.1 Taux de germination}

Par la méthode de papier Buvard décrite par ISTA, 2005 elle a consisté pour :

- Le taux de germination, à faire un trempage de 198 graines de chaque variétés dans une solution d'hypochlorite de sodium diluée à $2 \%$ pendant 5 min pour une désinfection de surface. Après trois rinçages dans de l'eau distillée stérilisée, ces graines ont été placées sur trois couches de papier Buvard. Puis, celles-ci ont été ensemencées de façon équidistante dans les boîtes de Pétri tapissées de 3 couches à raison de 06 graines par boîte. Une fois soumis à l'incubateur avec une température variant de 20 à $22^{\circ} \mathrm{C}$, les graines germées ont été dénombrées après 9 jours pour l'évaluation de la germination selon la formule:

$$
\% \text { de germination }=\frac{\text { Nombre de graines germées }}{\text { Nombre total des graines }} \times 100
$$




\section{III.3.2 Hauteur des plantes}

La mesure de la hauteur des plantes a été pris à l'aide d'une règle graduée le $45^{\mathrm{e}}, 52^{\mathrm{e}}, 59^{\mathrm{e}}$ et $66^{\mathrm{e}} \mathrm{JAS}$ sur un échantillon de 10 plants sélectionnés au hasard par unité élémentaire. Les mesures ont été faites sur les plantes se trouvant sur les billons intermédiaires et la hauteur est considérée comme la distance entre le collet et le bourgeon terminal de la plus grande plante.

\section{III.3.3 Nombres de feuilles}

Il a été déterminé par comptage direct des feuilles trifoliolées des différentes variétés sur les 10 plants sélectionnés comme échantillon en fonction des doses pour chaque bloc à partir le $45^{\mathrm{e}}, 52^{\mathrm{e}}, 59^{\mathrm{e}}$ et $66^{\mathrm{e}} \mathrm{JAS}$ pendant la phase végétative de la plante jusqu'à croissance standard des variétés.

\section{III.3.4 Précocité variétale}

L'évaluation de la précocité s'est basée sur le nombre de jours à la floraison et à la maturation des variétés. Le nombre de jour à la floraison a été pris pendant la période de floraison et celui de la maturité lorsque les plantes d'une unité élémentaire présentaient des gousses atteintes au stade mature pendant cette période. Il a été déterminé par comptage du nombre de jours écoulé de la mise en terre à la date où environ $50 \%$ de chaque unité expérimentale ont atteint la floraison et $70 \%$ des plants présentant des gousses à maturité.

\section{III.3.5 Rendement en gousse des variétés}

A maturité, le nombre de gousse par plants, le nombre total des gousses et le rendement en gousse par variétés en fonction des doses de mycorhizes ont été évaluées. Sur un échantillon de 10 plants choisis pour chaque variété, le nombre de gousse, la longueur des gousses en fonction des doses ont été déterminée.

\section{III.4 Analyse des données}

Les données collectées sur les paramètres étudiés ont été ordonnées et classées dans le tableur Excel de Microsoft. 2016. Ces données ont été transférées sur le logiciel SPSS. 21, pour analyse de la variance (ANOVA) et les moyennes ont été séparées à l'aide de Test de Tukey au seuil de probabilité $5 \%$.

\section{Résultats}

IV.1 Taux de germination des trois variétés de Voandzou au laboratoire Le Tableau 1 ci-dessous présente le taux de germination des semences des différentes variétés de voandzou après 9 jours d'observation. Il ressort de ce tableau que la variété Mendéo du Nord (V1M) présente un taux de 
germination $(95,45 \%)$ plus élevé que la variété locale (VL) de Dschang $(89,89$ $\%)$ et la variété Mendéo 2 (V2M) du Nord $(85,85 \%)$.

Tableau 1 : Taux de germination des différentes variétés Voandzou.

\begin{tabular}{cccc}
\hline Variétés & Graine étudiée & \% germination & Graine germée.boite $^{\mathbf{- 1}}$ \\
\hline VL & 198 & 89,89 & $5,39 \pm 0,86 \mathrm{a}$ \\
V1M & 198 & 95,45 & $5,73 \pm 0,51 \mathrm{a}$ \\
V2M & 198 & 85,85 & $5,15 \pm 0,94 \mathrm{a}$ \\
\hline
\end{tabular}

NB : VL = variété Locale Dschang, V1M = variété 1 Mendéo du Nord, V2M = variété 2 Mendéo du Nord

\section{IV.2 Hauteur des plantes}

La croissance en hauteur des plantes des trois variétés de Voandzou en fonction des différents traitements est présentée dans le Tableau 2. Il ressort de ce Tableau que la variation de la hauteur des plantes sous l'effet des différentes doses de mycorhize a augmentée pour les trois variétés, comparé aux plantes témoins. La dose D2 (20g) de mycorhize semble être plus favorable sur la croissance en hauteur des plantes pour les deux variétés du Nord Cameroun (V1M, V2M) et la variété Locale de Dschang (VL) durant le stade phénologique de la plante, par rapport la dose D1 (10g). Néanmoins cette dose (D1) s'avère plus efficace sur la hauteur des plantes à partir du $52^{\text {ème jour }}$ après semis sur les trois variétés (Tableau 2). Toutefois pendant le stade phénologique, la hauteur est plus importante avec l'application de la dose D1 de mycorhize pour la variété locale de Dschang (VL).

Tableau 2 : Hauteurs des plants selon les doses et variétés.

\begin{tabular}{cccccc}
\hline \multicolumn{5}{c}{ Hauteur des plants (cm) } \\
\hline Variété & Dose & $\mathbf{4 5}^{\mathrm{e}} \mathbf{J A S}$ & $\mathbf{5 2}^{\mathrm{e}} \mathbf{J A S}$ & $\mathbf{5 9}^{\mathrm{e}} \mathbf{J A S}$ & $\mathbf{6 6}^{\mathrm{e}} \mathbf{J A S}$ \\
\hline \multirow{2}{*}{ V1M } & D0 & $14,73 \pm 1,82 \mathrm{~b}$ & $14,08 \pm 2,21 \mathrm{c}$ & $17,91 \pm 2,75 \mathrm{c}$ & $19,08 \pm 2,63 \mathrm{bc}$ \\
& D1 & $14,07 \pm 1,88 \mathrm{~b}$ & $15,66 \pm 1,75 \mathrm{~b}$ & $19,31 \pm 2,19 \mathrm{c}$ & $20,32 \pm 4,56 \mathrm{bc}$ \\
& D2 & $16,44 \pm 1,43 \mathrm{a}$ & $16,34 \pm 2,37 \mathrm{ab}$ & $22,03 \pm 3,72 \mathrm{~b}$ & $22,40 \pm 2,68 \mathrm{~b}$ \\
\hline \multirow{3}{*}{ V2M } & D0 & $13,86 \pm 2,43 \mathrm{c}$ & $14,39 \pm 1,86 \mathrm{bc}$ & $16,58 \pm 3,98 \mathrm{c}$ & $17,33 \pm 3,93 \mathrm{c}$ \\
& D1 & $14,79 \pm 1,39 \mathrm{~b}$ & $15,32 \pm 1,99 \mathrm{~b}$ & $17,92 \pm 2,10 \mathrm{c}$ & $18,00 \pm 4,04 \mathrm{c}$ \\
& D2 & $15,79 \pm 1,48 \mathrm{ab}$ & $15,49 \pm 1,5 \mathrm{~b}$ & $18,71 \pm 2,27 \mathrm{c}$ & $18,23 \pm 5,46 \mathrm{c}$ \\
\hline \multirow{2}{*}{ VL } & D0 & $15,45 \pm 2,58 \mathrm{ab}$ & $16,99 \pm 2,69 \mathrm{a}$ & $23,4 \pm 3,31 \mathrm{ab}$ & $26,11 \pm 3,34 \mathrm{a}$ \\
& D1 & $15,13 \pm 1,89 \mathrm{ab}$ & $17,95 \pm 2,42 \mathrm{a}$ & $24,57 \pm 2,85 \mathrm{a}$ & $27,68 \pm 2,92 \mathrm{a}$ \\
& D2 & $16,14 \pm 2,66 \mathrm{a}$ & $17,49 \pm 2,56 \mathrm{a}$ & $24,29 \pm 3,50 \mathrm{ab}$ & $26,68 \pm 3,30 \mathrm{a}$ \\
\hline
\end{tabular}

NB: Les valeurs suivies de la même lettre dans la même colonne ne sont pas significativement différentes au seuil de $5 \%$ selon le Test de Tukey $(\mathrm{P} \leq 0.05)$. Les valeurs sont la moyenne et \pm Ecart-type. D0 = dose 0g, D1 = dose 10g, D2 = dose 20g, VL = variété

Locale Dschang, V1M = variété 1 Mendéo du Nord, V2M = variété 2 Mendéo du Nord

\section{IV.3 Nombre de feuilles}

Le nombre de feuilles des plantes des trois variétés de Voandzou en fonction des différents traitements est présenté dans le Tableau 3 ci-dessous. D'après ce Tableau, le nombre de feuilles des plantes pour les trois variétés a 
augmenté sous l'effet des deux doses de mycorhize comparé aux plantes témoins. Pour ce qui est des variétés du Nord Cameroun, seule la variété V1M parait plus favorable à la dose D1 $(10 \mathrm{~g})$ et la dose D2 $(20 \mathrm{~g})$ à partir du $52^{\mathrm{e}}$ au jour après semis. Par contre le nombre des feuilles la variété locale (VL) de

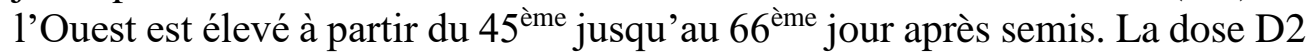
d'inoculum par apport à la dose D1 a eu un effet plus positif sur la densité du feuillage des plantes des deux variétés du Nord Cameroun (V1M et V2M).

Tableau 3 : Nombres de feuilles des plants selon les doses et variétés.

\begin{tabular}{cccccc}
\hline \multicolumn{5}{c}{ Nombre de feuilles } \\
\hline Variété & Dose & $\mathbf{4 5}^{\mathrm{e}} \mathbf{J A S}$ & \multicolumn{1}{c}{$\mathbf{5 2}^{\mathrm{e}} \mathbf{J A S}$} & \multicolumn{1}{c}{$\mathbf{5 9}^{\mathrm{e}} \mathbf{J A S}$} & $\mathbf{6 6}^{\mathrm{e}} \mathbf{J A S}$ \\
\hline \multirow{2}{*}{ V1M } & D0 & $11,06 \pm 2,98 \mathrm{a}$ & $17,67 \pm 4,91 \mathrm{ab}$ & $27,40 \pm 8,09 \mathrm{~b}$ & $32,86 \pm 10,32 \mathrm{ab}$ \\
& D1 & $12,66 \pm 2,27 \mathrm{a}$ & $17,80 \pm 4,94 \mathrm{ab}$ & $31,10 \pm 9,37 \mathrm{ab}$ & $34,50 \pm 11,43 \mathrm{ab}$ \\
& D2 & $11,73 \pm 2,37 \mathrm{a}$ & $21,33 \pm 4,58 \mathrm{a}$ & $35,66 \pm 6,32 \mathrm{a}$ & $39,60 \pm 7,60 \mathrm{a}$ \\
\hline \multirow{2}{*}{ V2M } & D0 & $13,10 \pm 3,17 \mathrm{a}$ & $18,56 \pm 4,19 \mathrm{ab}$ & $29,90 \pm 10,58 \mathrm{~b}$ & $36,30 \pm 10,66 \mathrm{ab}$ \\
& D1 & $12,06 \pm 3,62 \mathrm{a}$ & $16,00 \pm 4,54 \mathrm{~b}$ & $28,16 \pm 10,35 \mathrm{~b}$ & $33,56 \pm 12,34 \mathrm{ab}$ \\
& D2 & $10,96 \pm 2,69 \mathrm{a}$ & $16,63 \pm 4,87 \mathrm{~b}$ & $33,56 \pm 10,73 \mathrm{a}$ & $40,40 \pm 11,56 \mathrm{a}$ \\
\hline \multirow{2}{*}{ VL } & D0 & $8,50 \pm 2,16 \mathrm{~b}$ & $13,30 \pm 4,54 \mathrm{c}$ & $23,26 \pm 7,88 \mathrm{c}$ & $31,80 \pm 8,89 \mathrm{~b}$ \\
& D1 & $10,96 \pm 3,36 \mathrm{a}$ & $15,90 \pm 4,72 \mathrm{~b}$ & $27,90 \pm 7,71 \mathrm{bc}$ & $40,10 \pm 11,06 \mathrm{a}$ \\
& D2 & $8,60 \pm 2,26 \mathrm{~b}$ & $15,06 \pm 4,74 \mathrm{~b}$ & $26,66 \pm 9,04 \mathrm{~b}$ & $34,16 \pm 9,74 \mathrm{ab}$ \\
\hline
\end{tabular}

NB : Les valeurs suivies de la même lettre dans la même colonne ne sont pas significativement différentes au seuil de $5 \%$ selon le Test de Tukey $(\mathrm{P} \leq 0.05)$. Les valeurs sont la moyenne et \pm Ecart-type. $\mathbf{D 0}=$ dose $0 \mathrm{~g}, \mathbf{D 1}=$ dose $10 \mathrm{~g}, \mathbf{D 2}=$ dose $20 \mathrm{~g}, \mathbf{V L}=$ variété

Locale Dschang, V1M = variété 1 Mendéo du Nord, V2M = variété 2 Mendéo du Nord

\section{IV.4 Précocité variétale}

La Figure 3 nous montre la précocité variétale des différentes variétés de voandzou enregistrée du semis à la floraison des plants. On constate que le nombre de jours de la floraison et de la maturation varie selon l'espèce variétale (Figure 3). Ceci nous permet de déduire que, les deux variétés introduites (améliorées) du Nord Cameroun V1M et V2M sont des variétés précoces avec un nombre de jours de floraison de $40 \mathrm{JAS}$ et un cycle de maturation de 122 JAS (Figure 3). Par contre la variété VL de l'Ouest Cameroun est considérée comme étant une variété tardive avec un nombre de jours de floraison de 58 JAS et un cycle de maturation de 160 JAS (Figure 3). 


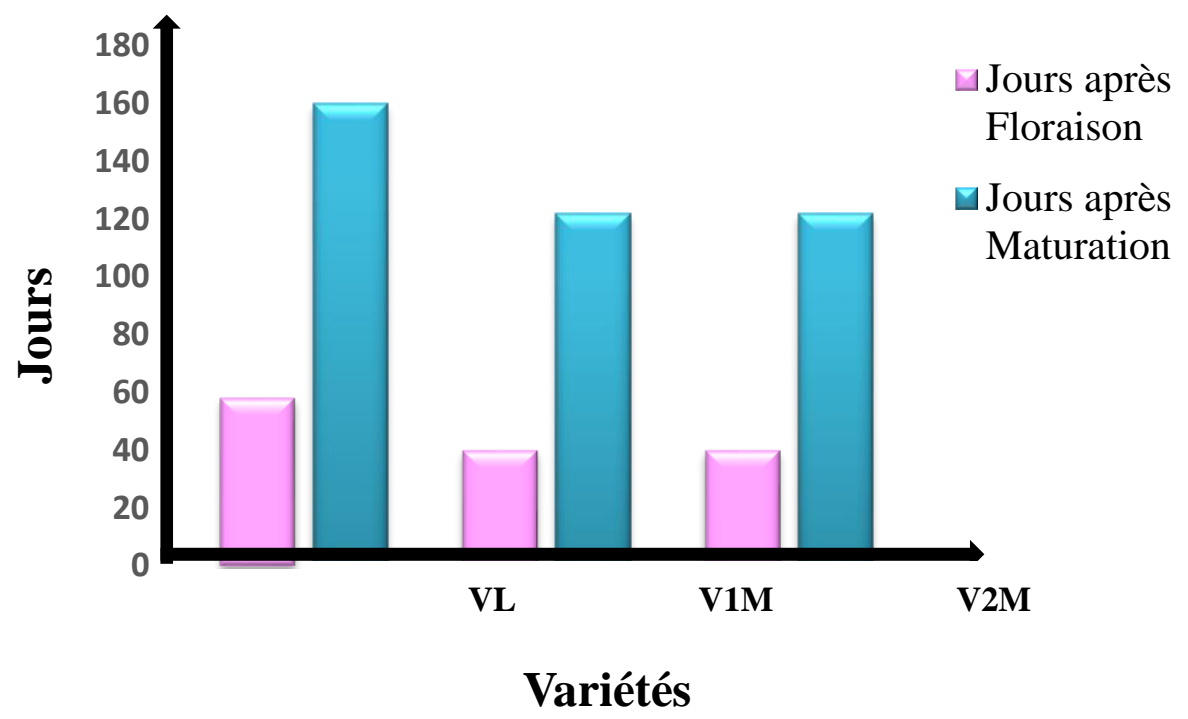

Figure 3 : Précocité des variétés

\section{IV.5 Rendements en gousses des variétés}

\section{IV.5.1 Effets des doses sur la longueur des gousses}

Les effets des différentes doses inoculées de mycorhize sur la longueur des gousses des différentes variétés de Voandzou sont présentés dans le Tableau 4 ci-dessous. On y observe de manière générale comparé aux témoins, que l'inoculation avec les doses D1 (10g) et D2 (20) a augmenté la longueur des gousses des différentes plantes de voandzou à l'exception des plantes de la variété V1M chez qui, l'inoculation avec les doses D1 et D2 n'a entrainé aucun changement positif sur la longueur des gousses. La longueur des gousses des plantes inoculées chez la variété V2M du Nord Cameroun est significativement supérieure $(\mathrm{P} \leq 0.05)$ à celle des plantes témoins. Par contre la dose D2 a plus favorisée la longueur des gousses de la variété locale (VL) de l'Ouest Cameroun (Tableau 4).

Tableau 4 : Longueur des gousses des variétés selon les traitements.

\begin{tabular}{cccc}
\hline & \multicolumn{4}{c}{ Longueur des gousses (cm). 10 plants $^{-1}$} \\
\hline Dose & VL & V1M & V2M \\
\hline D0 & $3,34 \pm 0,25 \mathrm{a}$ & $2,80 \pm 0,42 \mathrm{~b}$ & $2,68 \pm 0,5 \mathrm{~b}$ \\
D1 & $3,92 \pm 0,23 \mathrm{a}$ & $2,67 \pm 0,43 \mathrm{~b}$ & $3,06 \pm 0,21 \mathrm{a}$ \\
D2 & $4,14 \pm 0,22 \mathrm{a}$ & $2,77 \pm 0,46 \mathrm{~b}$ & $3,17 \pm 0,24 \mathrm{a}$ \\
\hline
\end{tabular}

NB : Les valeurs suivies de la même lettre dans la même colonne ne sont pas significativement différentes au seuil de $5 \%$ selon le Test de Tukey $(\mathrm{P} \leq 0.05)$. Les valeurs sont la moyenne et \pm Ecart-type. $\mathbf{D 0}=$ dose $0 \mathrm{~g}, \mathbf{D 1}=$ dose $10 \mathrm{~g}, \mathbf{D} 2=$ dose $20 \mathrm{~g}, \mathbf{V L}=$ variété

Locale Dschang, V1M = variété 1 Mendéo du Nord, V2M = variété 2 Mendéo du Nord 


\section{IV.5.2 Effets des doses sur le nombre de gousses}

Comparé aux plantes témoins, les différentes doses de mycorhize inoculées ont influencées de manière significative la production des gousses chez les plantes des trois variétés de Voandzou (Tableau 5). Avec la dose D1 $(10 \mathrm{~g})$ ou D2 (20g), Le nombre de gousse dénombré par plante est significativement supérieur chez les plantes inoculées comparé à celles non inoculées, sauf chez la variété $\mathrm{V} 1 \mathrm{M}$ du Nord Cameroun où l'analyse de variance montre un effet significatif $(P \leq 0.05)$ uniquement avec la dose D1 (Tableau 5).

Tableau 5 : Nombre de gousses par plante selon les doses et variétés.

\begin{tabular}{cccc}
\hline \multicolumn{3}{c}{${\text { Nombre de gousses.10 } \text { plants }^{-1}}^{-1}$} \\
\hline Dose & VL & V1M & V2M \\
\hline D0 & $13 \pm 2,84 \mathrm{c}$ & $13 \pm 5,41 \mathrm{c}$ & $9 \pm 4,18 \mathrm{~d}$ \\
D1 & $22 \pm 3,05 \mathrm{a}$ & $15 \pm 5,89 \mathrm{~b}$ & $12 \pm 2,94 \mathrm{c}$ \\
D2 & $22 \pm 2,63 \mathrm{a}$ & $14 \pm 4,8 \mathrm{c}$ & $11 \pm 1,97 \mathrm{c}$ \\
\hline
\end{tabular}

NB : Les valeurs suivies de la même lettre dans la même colonne ne sont pas significativement différentes au seuil de $5 \%$ selon le Test de Tukey $(\mathrm{P} \leq 0.05)$. Les valeurs sont la moyenne et \pm Ecart-type. D0 = dose $0 \mathrm{~g}, \mathbf{D} 1=$ dose $10 \mathrm{~g}, \mathbf{D} \mathbf{2}=$ dose $20 \mathrm{~g}, \mathbf{V L}=$ variété

Locale Dschang, V1M = variété 1 Mendéo du Nord, V2M = variété 2 Mendéo du Nord

\section{IV.5.3 Effets des doses sur le nombre total des gousses}

Le nombre total de gousse des plantes selon les traitements pour les trois variétés est présenté dans le Tableau 6. D'après ce tableau la variété VL de Dschang présente un nombre total de gousse significativement élevé compare aux plants témoin. Les plantes de la variété V1M du Nord Cameroun inoculées ont un nombre total de gousse plus élevé que chez les plantes non inoculées uniquement avec la dose D2 (20g) de mycorhize. Par contre, les plantes inoculées de la variété V2M du Nord Cameroun présentent un nombre total de gousse réduit par apport aux témoins avec les doses D1 $(10 \mathrm{~g})$ et D2 (20g) de mycorhize (Tableau 6).

Tableau 6 : Nombre total de gousses selon les doses et variétés.

\begin{tabular}{cccc}
\hline \multicolumn{3}{c}{ Nombre total des gousses } \\
\hline Dose & VL & V1M & V2M \\
\hline D0 & $465,33 \pm 65,65 \mathrm{~b}$ & $315,33 \pm 68,09 \mathrm{c}$ & $141,33 \pm 27,46 \mathrm{~d}$ \\
D1 & $622,67 \pm 75,59 \mathrm{a}$ & $252 \pm 85,03 \mathrm{~cd}$ & $123,67 \pm 51,43 \mathrm{~d}$ \\
D2 & $723 \pm 25,63 \mathrm{a}$ & $345,67 \pm 81,30 \mathrm{bc}$ & $115,33 \pm 61,01 \mathrm{~d}$ \\
\hline
\end{tabular}

NB : Les valeurs suivies de la même lettre dans la même colonne ne sont pas significativement différentes au seuil de $5 \%$ selon le Test de Tukey $(\mathrm{P} \leq 0.05)$. Les valeurs sont la moyenne et \pm Ecart-type. D0 = dose 0g, D1 = dose 10g, D2 = dose 20g, VL = variété

Locale Dschang, V1M = variété 1 Mendéo du Nord, V2M = variété 2 Mendéo du Nord 


\section{IV.5.4 Effets des doses sur le rendement des gousses}

Le Tableau 7 révèle le rendement moyen des gousses des trois variétés de Voandzou selon les traitements. Avec les doses D1 (10g) et D2 (20g), le rendement moyen des gousses est significativement plus grand chez les plantes inoculées de la variété VL de Dschang comparée aux plantes non inoculées (Tableau 7). Chez la variété V1M du Nord Cameroun, le rendement des gousses est significativement inferieur chez les plantes inoculées avec la dose D1 de mycorhize par apport au contrôle. Chez cette variété, le rendement le plus élevé a été enregistré avec la dose D2 (Tableau 7). Chez la variété V2M du Nord Cameroun par contre, aucune efficacité de la dose D1 et D2 sur le rendement des gousses n'a été relevée. Le rendement des gousses obtenues avec les deux doses pour cette variété sont significativement inferieur au rendement fourni par plants les témoins (Tableau 7). La comparaison des effets des deux doses de mycorhize, sur le rendement moyen des gousses n'est significative que chez la variété V1M du Nord Cameroun chez qui la dose D2 s'est avérée plus efficace (Tableau 7).

Tableau 7 : Rendement des gousses selon les doses et variétés.

\begin{tabular}{clll}
\hline & \multicolumn{3}{c}{ Rendement kg.ha $^{-1}$} \\
\hline Dose & \multicolumn{1}{c}{ VL } & \multicolumn{1}{c}{ V1M } & \multicolumn{1}{c}{ V2M } \\
\hline D0 & $1302 \pm 39,66 \mathrm{~b}$ & $1270,33 \pm 315,87 \mathrm{~b}$ & $622,33 \pm 232,67 \mathrm{~d}$ \\
D1 & $1630,66 \pm 81,39 \mathrm{a}$ & $994,67 \pm 330,74 \mathrm{c}$ & $437 \pm 181,51 \mathrm{e}$ \\
D2 & $1682,66 \pm 22 \mathrm{a}$ & $1599,67 \pm 381,6 \mathrm{a}$ & $537 \pm 284,19 \mathrm{e}$ \\
\hline
\end{tabular}

NB : Les valeurs suivies de la même lettre dans la même colonne ne sont pas significativement différentes au seuil de $5 \%$ selon le Test de Tukey $(\mathrm{P} \leq 0.05)$. Les valeurs sont la moyenne et \pm Ecart-type. $\mathbf{D 0}=$ dose $0 \mathrm{~g}, \mathbf{D} \mathbf{1}=$ dose $10 \mathrm{~g}, \mathbf{D} \mathbf{2}=$ dose $20 \mathrm{~g}, \mathbf{V L}=$ variété

Locale Dschang, V1M = variété 1 Mendéo du Nord, V2M = variété 2 Mendéo du Nord

\section{Discussions}

Dans le but d'évaluer les potentialités agronomiques des trois variétés, le pourcentage de germination a été estimé à 9 jours après semis comme recommande les travaux de Djé et al. (2005). Au cours de cette étude, nous avons observé un taux important de levée chez les trois variétés étudiées. Toutefois, aucune différence significative n'a été constatée entre les taux de germination des 3 variétés étudiées. Ces résultats peuvent s'expliquer non seulement par les meilleures conditions climatiques et environnementales favorisant la germination des semences des différentes variétés étudiées, mais aussi par l'aptitude à germer des graines des différentes variétés.

Dans les régions tropicales semi arides, il a été démontré que la symbiose mycorhizienne joue un rôle important dans le développement des plantes (Estaùn et al., 1997). Dans le but de mesurer l'effet des doses de mycorhizes sur la croissance végétative des variétés de voandzou, les données sur la hauteur des plants à $45^{\mathrm{e}}, 52^{\mathrm{e}}, 59^{\mathrm{e}}$ et $66^{\mathrm{e}} \mathrm{JAS}$ ont été collectées. Il ressort de nos résultats que, l'inoculation des CMA a induit une amélioration de la 
croissance en hauteur chez toutes les variétés du $45^{\mathrm{e}} \mathrm{JAS}$ jusqu'à la fin du cycle végétatif. D'après l'étude de Ogou et al. (2018) sur le Soja, l'infection mycorhizienne semble être effective à partir du $45^{\mathrm{e}} \mathrm{JAS}$ traduisant ainsi une différence de croissance en hauteur des plants mycorhizés et ceux non mycorhizés. Bien que statistiquement similaire, il existe une différence de 2 à $4 \%$ pour les variétés qui ont potentiellement réagit aux doses de mycorhizes sur la hauteur des plants inoculés et non inoculés chacun du $52^{\mathrm{e}}$ au $66^{\mathrm{e}} \mathrm{JAS}$. Ceci serait dû au fait qu'en cette phase de développement, l'on a un accroissement racinaire qui, en association symbiotique avec Glomus hoi créent un réseau de filaments dans le sol, permettant ainsi l'absorption de l'eau et des nutriments (Phosphore, Zinc, Cuivre, Molybdène) favorisant de ce fait la croissance de la plante d'une part, et d'autre part l'amplification des effets physiologiques complexes qui vont agir sur la hauteur des plants. Au cours de leurs travaux, Rajasekaran et Nagarajan (2005) ont également montré que l'apport d'inoculum de CMA en plein champ améliore la croissance de plusieurs espèces de Fabaceae et de Fourragères.

L'impact que pourrait avoir des traitements mycorhiziens sur le nombre de feuilles a aussi été déterminé. Les travaux de Laminou et al. (2009) ont montré que l'inoculation avec les mycorhizes ont un effet positif sur la biomasse totale des plants. Contrairement aux deux variétés Mendéo (V1M et V2M) du Nord Cameroun, seuls les résultats obtenus chez la variété VL de l'Ouest Cameroun nous permettent de dire de manière claire que, l'inoculation de mycorhizes pourrait induire l'augmentation de la densité du feuillage.

L'effet des doses de mycorhize sur la productivité en gousses a été évalué. Pour cela nous avons évalué la longueur et le nombre des gousses par plants, nous avons également évalué le nombre total et le rendement des gousses $\left(\mathrm{Kg}_{\mathrm{g}} \mathrm{ha}^{-1}\right)$. D'après nos résultats, l'inoculation des plants de Voandzou a permis d'augmenter de façon significative la longueur des gousses chez toutes les variétés. Selon Touil (2017), l'inoculation des plants par les souches de CMA via le phénomène d'endosymbiose influence sur la longueur et la largeur des gousses. Ces résultats sur l'inoculation arachidière aux différentes souches de CMA parmi lesquelles Glomus mosseae ont montrés un effet hautement positifs non seulement sur la longueur des gousses, mais aussi, ont agit nettement sur la largeur des gousses des plants. Cependant, la dose D2 (20g) est celle qui a eu le plus d'efficacité sur la longueur des gousses principalement chez les variétés locale de l'Ouest Cameroun (VL) et celle introduite V2M du Nord Cameroun. Cette différence pourrait s'expliquer par la bonne adhésion lors de l'inoculation en champ permettant une bonne formation des hyphes au niveau du système racinaire, qui mobilisent l'eau et solubilisent le Phosphore (Smith \& Read, 2008).

Nos résultats ont montré que, le nombre moyen de gousse a significativement augmenté chez les plants inoculés par rapport aux plants non 
inoculés. Avec la dose $10 \mathrm{~g}$ le nombre de gousse par plant est élevé chez toutes les variétés testées comparativement aux témoins respectifs des mêmes variétés. Avec la dose 20g, le nombre de gousse par plant est plus important uniquement chez la variété locale (VL) de l'Ouest Cameroun, comparé aux témoins. Par la même occasion nous avons observé une augmentation du nombre total des gousses des plants inoculés par rapport à ceux non inoculés. Avec les deux doses, le nombre total de gousse a été élevée chez la variété locale de l'Ouest comparé au témoin de la même variété. Nos résultats sont en adéquation avec ceux obtenu par Ogou et al. (2018). De plus Touil (2017) à travers ses travaux sur l'arachide a pu montrer que, l'interaction de plusieurs souches de CMA peut provoquer une augmentation significative du nombre de gousses et du poids des gousses par rapport aux plantes non inoculés. Toutefois le faible nombre de gousses enregistré chez certaines plantes peut avoir été occasionné par l'attaque non seulement par des rongeurs pendant la période de maturation des gousses, mais aussi par les nématodes en champ malgré la forte aptitude des mycorhizes à réduire le taux de multiplication des nématodes par le développement des arbuscules dans les tissus racinaires (Hauggaard-Nielson, 2005).

Avec l'utilisation des mycorhizes, et malgré les conditions pluviométriques insuffisantes aux exigences de Voandzou pendant la mise en place de notre essai (en contre saison), l'on enregistre une assez bonne productivité en gousse. Ce qui traduit la capacité qu'a les mycorhizes à aider les plantes à lutter contre la sécheresse (Smith \& Read, 2008). Comparer aux témoins, l'efficacité des mycorhizes sur le rendement en gousse n'a été révélée que chez les plantes de la variété locale VL de l'Ouest Cameroun avec les doses D1 et D2, et chez la variété introduite V1M du Nord Cameroun uniquement avec la dose D2. Ces résultats vont dans le même sens que ceux des travaux menés par Aboubacar et al. (2013) sur le Niébé et a montré que l'inoculation aux différentes souches améliore de manière significative le rendement en gousses du plant inoculé par rapport aux plants témoins. Les résultats obtenus avec les deux doses chez la variété VL de l'Ouest Cameroun pourrait s'expliquer par le fait que celle-ci est dans sa zone agro-écologique comparé aux variétés introduites du Nord Cameroun. Ce qui traduit l'expression de la potentialité de cette variété à l'inoculation aux différentes souches de mycorhize.

\section{Conclusion}

La présente étude, bien que déroulée en contre saison a montré que l'inoculation mycorhizienne en champ, s'est révélée plus ou moins efficace, dans la mesure où les résultats obtenus chez les plantes inoculées de la variété introduite V1M du Nord Cameroun et celles de la variété local VL de Dschang sont statistiquement différentes à ceux des plantes non inoculées des mêmes 
variétés. La variété introduite V1M du Nord Cameroun s'est avérée apte à s'implanter et s'adapter dans la zone d'étude. Son utilisation par les producteurs locaux pourrait donc être écologiquement rentable. La dose de mycorhize la plus souhaitable est la dose D2 (20g), permettant l'obtention d'un bon rendement. De plus, un travail de caractérisation des morphotypes disponible dans la région de l'Ouest Cameroun contribuerait à une meilleure valorisation de ces ressources.

\section{Remerciements}

Nous remercions l'Institut de Recherche Agricole pour le Développement (IRAD) de Garoua pour la production des semences améliorées. Nous remercions également la Faculté d'Agronomie et des Sciences Agricoles (FASA) pour avoir autorisé la mise : en place d'essai en champ à la Ferme d'Application et de la Recherche (FAR); les éventuelles manipulations au Laboratoire de Phytopathologie et de Zoologie Appliquée de la FASA.

\section{References:}

1. Aboubacar K, Ousmane M. Z, Amadou I. H, Issaka S, Zoubeirou M. A. (2013). Effet de la co-inoculation du rhizobium et de mycorhizes sur les performances agronomiques du niébé (Vigna unguiculata (L.) Walp.) au Niger. Journal of Applied Biosciences 72:5846-5854pp. ISSN 1997-5902.

2. Amadou H. I., Agali A., Sani D. O., Souleymane B. K. ( 2018). Variabilité Morphologique Et Agronomique Des Morphotypes De Voandzou (Vigna Subterranea (L.) Cultivés Dans La Zone Sahélienne Du Niger. Vol.14, No.36 ISSN: 1857-7881 (Print) e - ISSN 1857-7431.

3. Andriamananjara A. (2011). Système de culture à rotation voandzouriz pluvial sur les hautes terres de Madagascar. Rôle du voandzou (Vigna subterranea) sur la biodisponibilité de $\mathrm{P}$ dans les ferralsols. Résumé Thèse de Doctorat, Ecole Supérieure des Sciences Agronomiques, Université d'Antananarivo, Madagascar.1-321pp.

4. Bado B. V. (2002). Thèse sur: Rôle des légumineuses sur la fertilité des sols ferrugineux tropicaux des zones guinéenne et soudanienne du Burkina faso.183pp.

5. BAMNET/IPGRI/IITA. (2000). Descripteur du pois bambara (Vigna subterranea). Institut international des ressources phytogénétiques, Rome, Italie; Institut International d'Agriculture Tropicale, Ibadan, Nigeria; Réseau International de pois bambara, Allemagne. 48pp.

6. Bowers J. H., Sanogo S., Tondje P. R., Hebbar P. K., Lumsden R. D. (2001). Developing strategies for biological control of black pod, monilia pod rot, and witches'broom on Cacao. In : Proceedings of the 
1st west and central Africa training workshop on biocontrol of plant diseases, with special reference to cacao black pod diseases. 25-29 june, 2001. Douala (Cameroun).10-16pp.

7. Ciampalini, R., Billi, P., Ferrari, G., Borselli, L., Follain S. (2011). Soil erosion induced by land use changes as determined by plough marks and field evidence in the Aksum area (Ethiopia). Agriculture, Ecosystems and Environment 146 (2012) 197-208pp.

8. Dalgaard T., Hutchings, N. J., Porter, J. R. (2003). Agroecology, scaling and interdisciplinarity. Agricultural, Ecosystems, Environment, 100: 39-51pp. Dinkelaker, B., Rtimhe1d, V.

9. Dalgaard, T., Hutchings, N. J., Porter, J. R. (2003). Agroecology, scaling and interdisciplinarity. Agricultural, Ecosystems, Environment, 100: 39-51pp. Dinkelaker, B., Rtimhe1d, V.

10. Diallo K. S., Koné K. Y., Soro D., Assidjo N. E., Yao K. B., Gnakri D. (2015). Caracterisation biochimique et fonctionnelle des graines de sept cultivars de voandzou [vigna subterranea (1.) verdc. fabaceae] cultives en côte d'ivoire. edition vol.11, No.27 ISSN: 1857 - 7881 (Print) e - ISSN 1857- 7431.

11. Djè Y., Bonny B. S., Bi I. A. Z. (2005). Observations préliminaires de la variabilité entre quelques morphotypes de voandzou (Vigna subterranea L. Verdc., Fabaceae) de Côte d'Ivoire. Biotechnologie, Agronomy, Société et Environnement, 9: 1-16pp.

12. Estaùn V., Save R., Biel C. (1997). AM inoculation as a biological tool to improve plant revegetation of a disturbed soil with Rosmarinus officinalis under semi-arid conditions. Applied Soil Ecology, 6, 223229. Gange A. C et Ayres R. L (1999). On the relation between arbuscular mycorrhizal colonisation and plant benefit. Oikos, 87, 615 $-621 \mathrm{pp}$.

13. Hauggaard-Nielson, 2005. Intercropping of Wheat and Pea as Influenced by Nitrogen Fertilization. Nutrient Cycling in Agroecosystems., 73 (2): 201:201-21273. Doi: 10.1007/s10705-0052475-9.

14. IRAD. (2002). Rapport d'activités. Données pluviométriques de l'année 2002.

15. ISTA. (2005). International Rules for Seed Testing. Edition 2005. International Seed Testing Association, Bassersdorf, Suisse.

16. Laminou M. O., Ibrahim, D., Campanella B., Paul R. (2009). Effets de l'inoculation mycorhizienne du substrat sur la croissance et la résistance au stress hydrique de cinq espèces fixatrices de dunes : Acacia raddiana Savi ; Acacia nilotica (L.) Willd. Ex Del. var. adansonii ; Acacia senegal (L.) Willd ; Prosopis chilensis Stunz. et Bauhinia rufescens Lam. Geo-Eco-Trop., 33, n.s.: 115-124pp. 
17. Linnemann A. R., Westphal E., Wessel M. (1995). Photoperiod regulation of development and growth in Bambara groundnut (Vigna subterranea). Field Crops Research, 40: 39-47pp.

18. Madou C., Watching D., Vatsou J., Ardjoune F., Ndjouenkeu R., Goudoum A., Ngassoum M. B., Ngamo T. S. L. (2018). Pratiques Paysannes De Production Durable Des Graines De Voandzou [Vigna Subterranea (L.) Verdc.] Pour La Sécurité Alimentaire Dans Le Cameroun Septentrional. Vol.14, No.18 ISSN: 1857 - 7881 (Print) e ISSN 1857- 7431.

19. Manga M. A., Akoa A., Abolo D., Mbang A. A., Bedimo J. A. M., Nomo L. E. B., Akume N. D. (2013). Structure et composition floristiques des agro-forêts à base de caféiers arabica (Coffea arabica L.) dans les hauts plateaux de l'Ouest du Cameroun. International Journal of Biological and Chemical Sciences, 7 (4): 1474-1489pp.

20. Mekuriaw A., Heinimann A., Zeleke G., Hurni H. (2017). Factors influencing the adoption of physical soil and water conservation practices in the Ethiopian highlands. International Soil and Water Conservation Research 6 (2018) 23-30pp.

21. Minka S. R., Bruneteau M. (2000). Partial chemical composition of Bambara pea (Vigna subterranea L. Verdc). Food Chemistry, 68: 273276pp.

22. Moges, D. M., Taye, A. A. (2017). Determinants of farmers' perception to invest in soil and water conservation technologies in the North-Western Highlands of Ethiopia. International Soil and Water Conservation Research 5 (2017) 56-61pp.

23. Nacoulma-Ouédraogo O. (1996). Plantes médicinales et pratiques traditionnelles au Burkina-Faso : cas du plateau central. Thèse de Doctorat ès Sciences Naturelles, Université de Ouagadougou, Burkina-Faso.

24. Ogou A., Tchabi A., Tounou A. K., Agboka K., Sokame B. M. (2018). Effet de quatre souches de champignons Mychoriziens arbusculaires sur Meloidogyne spp., principal nématode parasitaire du soja (Glycine max (L.)) au Togo. Journal of Applied Biosciences 127: 12758-12769 ISSN 1997-5902.

25. Onimawo I. A., Momoh A. H., Usman A. (1998). Proximate composition and functional properties of four cultivars of Bambara groundnut (Voandzeia subterranea). Plant Foods for Human Nutrition 53, 153-158pp.

26. Oparaeke A. M. and Bunmi J. O. (2006). Bioactivity of two podered spices (Piper Guineense) (Dunal) A. Richard) as home masses insecticides against Callosobruchus subinnotatus (pic) on stored 
Bambara groundnut. In: Agricultura tropical and subtropical, 39(2): 132-133pp.

27. Ouoba A., Nadembega S., Konaté M. N., Hervé Nandkangré., Ouédraogo M., Sawadogo M. (2018).Variabilité de la coloration du tégument des grains de voandzou cultivées au Burkina Faso et les noms locaux associés. Vol.14, No.33 ISSN: 1857 - 7881 (Print) e ISSN 1857- 7431.

28. Rajasekaran S., Nagarajan S. M. 2005. Effect of dual inoculation (AM fungi and Rhizobium) on chlorophyll content of Vigna unguiculata (L). Walp. var. Pusa 151. Mycorrhiza News. 17: 10-11pp.

29. Rapport Projet C2D/ PROGRAMME D'APPUI A LA RECHERCHE AGRONOMIQUE. (2013). Contribution de la recherche à l'amélioration de la production et la consommation des légumineuses alimentaires au Cameroun. 57pp.

30. Smith S. E and Read D. J. (2008). Mycorrhizal symbiosis. Third Edition, London, UK, Academic press; 787pp.

31. Touil W. (2017). Effets comparés des champignons mycorhiziens arbusculaires et des Rhizobia isolés d'un sol algérien avec ceux du commerce, sur le rendement de l'arachide Arachis hypogaea (L.).1$169 \mathrm{pp}$. Thèse de doctorat de l'université de badji mokhtar-annaba. Discipline ou spécialité : Biosciences Végétales. 